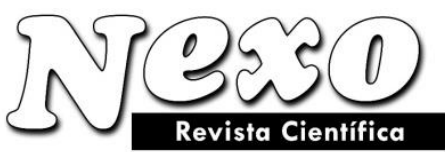

Vol. 33, No. 01, pp. 01-21/Junio 2020

\title{
EVALUACIÓN DE UN INTERCAMBIADOR DE CALOR DE PLACAS PARA EL ENFRIAMIENTO DE ÁCIDO ACÉTICO
}

\section{EVALUATION OF A PLATE HEAT EXCHANGER FOR ACETIC ACID COOLING}

\author{
A. Pérez Sánchez 1,*, E.J. Pérez Sánchez², E. Ranero Gonzalez ${ }^{1}$ \\ ${ }^{1}$ Universidad de Camagüey. Facultad de Ciencias Aplicadas. Camagüey, Cuba. \\ ${ }^{2}$ Empresa Servicios Automotores S.A., Dirección Comercial, Ciego de Ávila, Cuba. \\ *amauryps@nauta.cu
}

(recibido/received: 18-agosto-2019; aceptado/accepted: 19-octubre-2019)

\begin{abstract}
RESUMEN
En las aplicaciones de intercambio de calor actuales, el intercambiador de calor de placas (ICP) es muy empleado en las industrias químicas y la alimenticia. En el presente trabajo se efectuó la evaluación de un ICP para llevar a cabo el enfriamiento de una corriente de ácido acético, utilizando etilenglicol como agente refrigerante. Se obtuvo un valor del porcentaje de sobrediseño de superficie (PSS) de 29,34\%, una efectividad de transferencia de calor $(\varepsilon)$ de 0,667 , un número de unidades de transferencia (NUT) de 1,316, mientras que las caídas de presión de tanto el ácido acético $(6,70 \mathrm{kPa})$ como el etilenglicol $(82,06$ $\mathrm{kPa})$ se encontraron por debajo del límite máximo permisible por el proceso $(100 \mathrm{kPa})$. El incremento del caudal de alimentación del ácido acético aumentó los valores de los tres parámetros considerados, mientras que el incremento de la temperatura de entrada del ácido acético aumentó tres de las variables tomadas en cuenta, con excepción de la caída de presión del ácido acético, que se redujo. El ICP propuesto puede ser empleado satisfactoriamente para el proceso de transferencia de calor evaluado.
\end{abstract}

Palabras claves: Ácido acético; Caída de presión; Efectividad; Evaluación; Intercambiador de calor de placas.

\section{ABSTRACT}

In today heat exchange applications, the plate heat exchanger (PHE) is quite used in chemical en food industries. At the present work the assessment of a PHE to carry out the cooling of an acetic acid stream, using ethylene glycol as refrigerant agent was accomplished. It was obtained a value of $29.34 \%$ for the percent over surface design (OS), a heat exchanger effectiveness $(\varepsilon)$ value of 0.667 , a number of heat transfer units (NTU) value of 1.316, while the pressure drop results for both the acetic acid $(6.70 \mathrm{kPa})$ and ethylene glycol $(82.06 \mathrm{kPa})$ were below the maximum permissible limit for the process $(100 \mathrm{kPa})$. The increment of the acetic acid feed flowrate increased the values of the three parameters considered, while the increment of the acetic acid inlet temperature augmented three of the variables taken into account, except for the acetic acid pressure drop, which was reduced. The proposed PHE can be satisfactorily used for the evaluated heat transfer process.

Keywords: Acetic acid, Pressure drop, Effectiveness, Assessment, Plate heat exchanger.

Nexo Revista Científica / Vol. 33, No. 01, pp. 01-21 / Junio 2020 


\section{A. Pérez Sánchez et al.}

\section{NOMENCLATURA}

$\mathrm{A}_{1}$

$\mathrm{A}_{1 \mathrm{p}}$

$\mathrm{A}_{\mathrm{e}}$

$\mathrm{A}_{\mathrm{f}}$

b

C

$\mathrm{C}^{*}$

$\mathrm{C}_{\mathrm{h}}$

$\mathrm{Cp}$

$\mathrm{D}_{\mathrm{h}}$

$D_{p}$

$\mathrm{e}_{\mathrm{p}}$

f

FL

Fs

G

$\mathrm{G}_{\mathrm{p}}$

$\mathrm{h}$

$\mathrm{k}$

$\mathrm{k}_{\mathrm{p}}$

$\mathrm{K}_{\mathrm{p}}$

$\mathrm{L}_{\mathrm{a}}$

$\mathrm{L}_{\mathrm{ef}}$

$\mathrm{L}_{\mathrm{h}}$

$\mathrm{L}_{\mathrm{p}}$

$\mathrm{L}_{\mathrm{v}}$

$\mathrm{m}$

$\mathrm{m}$

$\mathrm{m}_{\mathrm{c}}$

n

$\mathrm{N}_{\mathrm{cp}}$

$\mathrm{N}_{\mathrm{e}}$

$\mathrm{N}_{\mathrm{p}}$

$\mathrm{N}_{\mathrm{t}}$

NUT

$\mathrm{p}$

Pr

PSS

$\Delta \mathrm{P}^{\prime}$

$\Delta \mathrm{P}_{\mathrm{f}}$

$\Delta \mathrm{P}_{\mathrm{p}}$

$\Delta \mathrm{P}_{\mathrm{t}}$

$\mathrm{Q}$

$\mathrm{R}$

$\operatorname{Re}$
Área de transferencia de calor de una placa simple Área proyectada de la placa

Área total efectiva

Área de flujo de un canal

Abertura promedio del canal de flujo

Tasa de capacidad calórica

Relación de la tasa de capacidad calórica

Parámetro de uso en las ecuaciones (24) y (25)

Calor especifico

Diámetro equivalente hidráulico del canal

Diámetro de todos los puertos

Espesor de las placas

Factor de fricción

Factor de limpieza

Factor de seguridad

Velocidad másica

Velocidad másica del puerto

Coeficiente pelicular de transferencia de calor

Conductividad térmica

Conductividad térmica del material de las placas

Parámetro de uso en las ecuaciones (33) y (34)

Amplitud efectiva del canal

$\mathrm{kg} / \mathrm{m}^{2} . \mathrm{s}$

$\mathrm{kg} / \mathrm{m}^{2} . \mathrm{s}$

$\mathrm{W} / \mathrm{m}^{2} \cdot \mathrm{K}$

$\mathrm{W} / \mathrm{m} . \mathrm{K}$

$\mathrm{W} / \mathrm{m} \cdot \mathrm{K}$

$\mathrm{m}$

$\mathrm{m}$

$\mathrm{m}$

$\mathrm{m}$

$\mathrm{m}$

$\mathrm{kg} / \mathrm{s}$

$\mathrm{kg} / \mathrm{s}$

Parámetro de uso en las ecuaciones (24) y (25)

Número de canales por pase

Número efectivo de placas

Número de pases

Número total de placas

Número de Unidades de Transferencia

Paso de la placa

$\mathrm{m}$

Número de Prandtl

Porcentaje de sobrediseño de superficie $\%$

Caída de presión permisible $\quad \mathrm{Pa}$

Caída de presión friccional $\quad \mathrm{Pa}$

Caída de presión en los conductos del puerto $\quad \mathrm{Pa}$

Caída de presión total $\quad \mathrm{Pa}$

Carga de calor

$\mathrm{W}$

Factor de ensuciamiento

$\mathrm{m}^{2} \cdot \mathrm{K} / \mathrm{W}$

Número de Reynolds

Nexo Revista Científica / Vol. 33, No. X1, pp. 01-21 / Junio 2020 


$\begin{array}{cc}\text { Temperatura del fluido frío } & { }^{\circ} \mathrm{C} \\ \text { Temperatura del fluido caliente } & { }^{\circ} \mathrm{C} \\ \text { Temperatura media del fluido frío } & { }^{\circ} \mathrm{C} \\ \text { Temperatura media del fluido caliente } & { }^{\circ} \mathrm{C} \\ \text { Diferencia media verdadera de temperatura } & { }^{\circ} \mathrm{C} \\ \text { Coeficiente global de transferencia de calor } & \mathrm{W} / \mathrm{m}^{2} . \mathrm{K} \\ \text { Símbolos griegos } & \\ \text { Ángulo de la placa Chevron } & { }^{\circ} \\ \text { Efectividad de transferencia de calor } & \\ \text { Densidad } & \mathrm{kg} / \mathrm{m}^{3} \\ \text { Factor de expansión } & \\ \text { Viscosidad } & \mathrm{Pa} . \mathrm{s} \\ \text { Subíndices } & \\ \text { Entrada } & \\ \text { Salida } & \\ \text { Fluido caliente } & \\ \text { Fluido frío } & \\ \text { Limpio } & \\ \text { Sucio } & \end{array}$

\section{INTRODUCCIÓN}

Un intercambiador de calor es un sistema termodinámico el cual es más comúnmente usado en la industria de procesos para intercambiar energía calórica entre los fluidos fluyendo en la misma dirección o de forma opuesta. Es deseable que la efectividad del intercambiador de calor se mantenga lo más elevada posible. El rendimiento de un intercambiador de calor puede mejorarse mediante la adición de aletas o corrugaciones (Singh \& Jakhar, 2014).

Los intercambiadores de calor de placas (ICP) constituyen unos de los equipos de transferencia de calor más eficientes desde el punto de vista térmico que se disponen en la actualidad. Entre sus ventajas se encuentran su elevada compactación, su bajo costo, menor ensuciamiento, flexibilidad para modificar el área de transferencia de calor, facilidad de mantenimiento, capacidad multifase, expansibilidad y un área de transferencia de calor elevada (Arsenyeva et al., 2011; Kumbhare \& Dawande, 2013).

El intercambiador de placas consiste de un paquete de placas metálicas corrugadas equipadas con juntas, presionadas entre sí en un marco. Los fluidos fluyen a través de una seria de canales de flujo en paralelo e intercambian calor a través de las placas delgadas metálicas corrugadas. El diseño de las juntas y los puertos cerrados de las placas determinan el arreglo de flujo de los fluidos, el cual puede ser en paralelo, en serie o una de las varias combinaciones posibles de los dos. La distribución de flujo, el número de placas, el tipo de junta y la localización de la alimentación caracterizan la configuración del intercambiador (Pinto \& Gut, 2002).

Los ICP se emplean para servicios con temperaturas y presiones moderadas, y para fluidos inocuos. Estas son las condiciones usualmente encontradas en las industrias de bebidas y de alimentos, mientras que la facilidad de limpieza explica la amplia aceptación de este equipo en tales aplicaciones (Cao, 2010).

El método efectividad-Número de Unidades de Transferencia (NUT) se emplea para intercambiadores de calor cuando se desconocen las temperaturas de salida de los fluidos caliente y frío, así como también la tasa de transferencia de calor desde el fluido caliente hacia el frio. En este caso, se conocen las

Nexo Revista Científica / Vol. 33, No. 01, pp. 01-21 / Junio 2020 


\section{A. Pérez Sánchez et al.}

dimensiones del intercambiador de calor. Un ejemplo de un caso donde este método puede utilizarse es cuando no se está empleando un intercambiador de calor, y se desea conocer si este equipo puede ser empleado para una determinada aplicación, esto es, si su rendimiento soportará las especificaciones requeridas del proceso (Pisinger, 2018).

Son varios los autores que han estudiado o evaluado ICP. En este sentido (Opatril et al., 2016) presentaron un método de evaluación de un ICP basado en datos experimentales y el método gráfico de Wilson, con el fin de obtener el coeficiente de transferencia de calor y la caída de presión entre la entrada y salida de los fluidos. También (Pinto \& Gut, 2002) propusieron un método de optimización para determinar la mejor configuración de un ICP, con el objetivo de seleccionar la configuración con la mínima área de transferencia de calor que aún satisfaga restricciones tales como el número de canales, la caída de presión de ambos fluidos, las velocidades del canal de flujo y la efectividad térmica del equipo.

Además, (Varona et al., 2007) analizaron los parámetros técnicos y operacionales relacionados con el mal funcionamiento de un ICP utilizado para el enfriamiento de mosto en una fábrica de cervezas, efectuando para ello análisis experimentales y aplicando además una metodología de cálculo para la evaluación térmica e hidrodinámica del intercambiador. Por otro lado, (Arsenyeva et al., 2009) efectuaron el diseño óptimo de un ICP multi-paso con agrupamiento mezclado de placas, con el fin de formular un problema matemático el valor mínimo de funciones objetivo discretas/continuas no lineales implícitas, seleccionando como variables de optimización el número de pases para ambas corrientes, el número de placas con diferentes geometrías de corrugación en cada paso, y el tipo y dimensión de la placa. El método de optimización fue posteriormente implementado en forma de software para efectuar los cálculos requeridos. En otro estudio, (Sreejith et al., 2014) diseñaron un ICP con el fin de determinar el coeficiente global de transferencia de calor, la tasa de transferencia de calor y el número de placas. También se realizó una optimización del costo del equipo. Torres y col. (Torres et al., 2016) efectuaron una investigación en un ICP con el objetivo de determinar los coeficientes de transferencia de calor y la influencia de las incrustaciones en la perdida de eficiencia de la instalación. Mediante el empleo de un procedimiento iterativo se determinó la ecuación del número de Nusselt y su dependencia con los numero de Reynolds y Prandtl, aplicando para ello un diseño experimental multifactorial, asi como también la realización de mediciones de los parámetros de trabajo de la instalación en función del tiempo. Por último, (Singh \& Jakhar, 2014) efectuaron una investigación experimental para calcular el coeficiente global de transferencia de calor y el factor de ensuciamiento de un ICP, evaluando además el factor de limpieza para predecir la próxima fecha de limpieza del equipo.

En el presente trabajo se realiza la evaluación de un ICP propuesto para efectuar el enfriamiento de una corriente de ácido acético, determinando parámetros de importancia tales como el porcentaje de sobrediseño de superficie (PSS), la caída de presión de cada fluido, la efectividad de transferencia de calor del intercambiador $(\varepsilon)$ y el número de unidades de transferencia (NUT). También se evalúa la influencia del incremento de dos variables de la corriente de ácido acético, las cuales son caudal másico $\left(\mathrm{m}_{\mathrm{C}}\right)$ y temperatura de entrada $\left(\mathrm{T}_{1}\right)$, sobre determinados parámetros de diseño y operación del equipo.

\section{MATERIALES Y MÉTODOS}

\subsection{Definición del problema}

Se requiere de un intercambiador de calor para enfriar $24 \mathrm{~kg} / \mathrm{s}$ de una corriente de ácido acético desde 80 ${ }^{\circ} \mathrm{C}$ hasta $30{ }^{\circ} \mathrm{C}$, utilizando etilenglicol a $5{ }^{\circ} \mathrm{C}$ cuya temperatura de salida no puede exceder $\operatorname{los} 25^{\circ} \mathrm{C}$ (Figura 1). La caída de presión máxima permisible para ambas corrientes no debe superar los $100 \mathrm{kPa}$. Se propone utilizar un ICP equipado con 107 placas del tipo Chevron, teniendo una amplitud efectiva del canal de $63 \mathrm{~cm}$, un diámetro del puerto de $0,2 \mathrm{~m}$, una distancia vertical entre puertos de 1,55 m, un espesor de la placa de $0,7 \mathrm{~mm}$, un ángulo de Chevron de $50^{\circ}$, un factor de expansión de 1,25 , un área total efectiva

Nexo Revista Científica / Vol. 33, No. X1, pp. 01-21 / Junio 2020 
de $115 \mathrm{~m}^{2}$, una longitud del paquete de placas comprimido de $0,44 \mathrm{~m}$ y una distancia horizontal entre puertos de $0,43 \mathrm{~m}$. Las placas son de acero inoxidable $316(\mathrm{k}=16,5 \mathrm{~W} / \mathrm{m} . \mathrm{K})$. Para un arreglo a contracorriente del tipo un paso/un paso, determine si el intercambiador de calor propuesto es factible de utilizar en la operación.

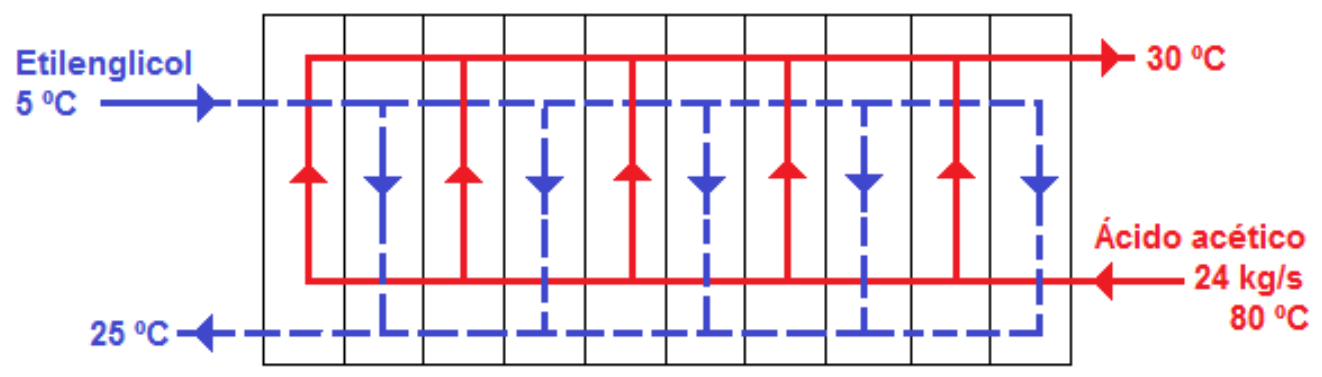

Fuente: Elaboración propia

Figura 1. Representación esquemática de las condiciones de operación

\subsection{Metodología de cálculo a emplear}

Para darle solución al problema se hará uso de la metodología de cálculo publicada en (Kakaç et al., 2012), en la cual se obtiene como resultados principales el porcentaje sobrediseño de superficie (PSS) del intercambiador de calor, la caída de presión de cada corriente, la efectividad de transferencia de calor del intercambiador $(\varepsilon)$ y el número de unidades de transferencia (NUT). A continuación de muestran cada uno de los pasos a seguir para efectuar la evaluación del equipo propuesto.

\subsubsection{Porcentaje de sobrediseño de la superficie (PSS)}

Paso 1. Recopilación de los datos iniciales de las corrientes

\begin{tabular}{lccc}
\hline Parámetro & Fluido caliente & Fluido frío & Unidades \\
\hline Caudales másicos & $\mathrm{m}_{\mathrm{C}}$ & $\mathrm{m}_{\mathrm{f}}$ & $\mathrm{kg} / \mathrm{s}$ \\
Temperatura de entrada & $\mathrm{T}_{1}$ & $\mathrm{t}_{1}$ & ${ }^{\circ} \mathrm{C}$ \\
Temperatura de salida & $\mathrm{T}_{2}$ & $\mathrm{t}_{2}$ & ${ }^{\circ} \mathrm{C}$ \\
Caída de presión máxima permisible & $\Delta \mathrm{P}^{\prime}{ }_{(\mathrm{c})}$ & $\Delta \mathrm{P}^{\prime}(\mathrm{f})$ & $\mathrm{Pa}$ \\
Factor de ensuciamiento & $\mathrm{R}_{\mathrm{C}}$ & $\mathrm{R}_{\mathrm{f}}$ & $\mathrm{m}^{2} \cdot \mathrm{K} / \mathrm{W}$ \\
\hline Fuente: Elaboración propia
\end{tabular}

Paso 2. Recopilación de los datos iniciales del intercambiador de calor de placas

\begin{tabular}{lcc}
\hline Parámetro & Símbolo & Unidades \\
\hline Espesor de las placas & $\mathrm{e}_{\mathrm{p}}$ & $\mathrm{m}$ \\
Ángulo de la placa Chevron & $\beta$ & $\mathrm{o}$ \\
Número total de placas & $\mathrm{N}_{\mathrm{t}}$ & - \\
Factor de expansión & $\phi$ & - \\
Número de pases & $\mathrm{N}_{\mathrm{p}}$ & - \\
Área total efectiva & $\mathrm{A}_{\mathrm{e}}$ & $\mathrm{m}^{2}$ \\
Diámetro de todos los puertos & $\mathrm{D}_{\mathrm{p}}$ & $\mathrm{m}$ \\
Longitud del paquete de placas comprimido & $\mathrm{L}_{\mathrm{p}}$ & $\mathrm{m}$ \\
Distancia vertical entre puertos & $\mathrm{L}_{\mathrm{v}}$ & $\mathrm{m}$ \\
Distancia horizontal entre puertos & $\mathrm{L}_{\mathrm{h}}$ & $\mathrm{m}$ \\
Amplitud efectiva del canal & $\mathrm{L}_{\mathrm{a}}$ & $\mathrm{m}$ \\
Conductividad térmica del material de las placas & $\mathrm{k}_{\mathrm{p}}$ & $\mathrm{W} / \mathrm{mK}$ \\
\hline
\end{tabular}

Nexo Revista Científica / Vol. 33, No. 01, pp. 01-21 / Junio 2020 


\section{A. Pérez Sánchez et al.}

Paso 3. Cálculo de la temperatura media de cada corriente:

Fluido caliente:

$$
\bar{T}=\frac{T_{1}+T_{2}}{2}
$$

Fluido frío:

$$
\bar{t}=\frac{t_{1}+t_{2}}{2}
$$

Paso 4. Búsqueda de las propiedades físicas de los fluidos a la temperatura media determinada en el paso anterior:

\begin{tabular}{lccc}
\hline Propiedad & Fluido caliente & Fluido frío & Unidades \\
\hline Calor especifico & $\mathrm{Cp}_{\mathrm{C}}$ & $\mathrm{C} \mathrm{p}_{\mathrm{f}}$ & $\mathrm{J} / \mathrm{kg} \cdot \mathrm{K}$ \\
Viscosidad & $\mu_{\mathrm{C}}$ & $\mu_{\mathrm{f}}$ & Pa.s \\
Conductividad térmica & $\mathrm{k}_{\mathrm{C}}$ & $\mathrm{k}_{\mathrm{f}}$ & $\mathrm{W} / \mathrm{m} \cdot \mathrm{K}$ \\
Densidad & $\rho_{\mathrm{C}}$ & $\rho_{\mathrm{f}}$ & $\mathrm{kg} / \mathrm{m}^{3}$ \\
\hline
\end{tabular}

Paso 5. Cálculo de la carga de calor (Q):

Fluido caliente:

$$
Q=m_{C} \cdot C p_{C} \cdot\left(T_{1}-T_{2}\right)
$$

Fluido frío:

$$
Q=m_{f} \cdot C p_{f} \cdot\left(t_{2}-t_{1}\right)
$$

Paso 6. Diferencia media verdadera de temperatura para flujo a contracorriente $\left(\Delta \mathrm{T}_{\mathrm{m}}\right)$ :

$$
\Delta T_{m}=\frac{\left(T_{1}-t_{2}\right)-\left(T_{2}-t_{1}\right)}{\ln \frac{\left(T_{1}-t_{2}\right)}{\left(T_{2}-t_{1}\right)}}
$$

Paso 7. Número efectivo de placas $\left(\mathrm{N}_{\mathrm{e}}\right)$ :

$$
N_{e}=N_{t}-2
$$

Paso 8. Longitud de flujo efectiva entre los puertos verticales $\left(\mathrm{L}_{\mathrm{ef}}\right)$ :

$$
L_{e f} \approx L_{v}
$$

Paso 9. Paso de la placa (p):

$$
p=\frac{L_{p}}{N_{t}}
$$

Paso 10. Abertura promedio del canal de flujo (b): 


$$
b=p-e_{p}
$$

Paso 11. Área de flujo de un canal $\left(\mathrm{A}_{f}\right)$ :

$$
A_{f}=b \cdot L_{a}
$$

Paso 12. Área de transferencia de calor de una placa simple $\left(\mathrm{A}_{1}\right)$ :

$$
A_{1}=\frac{A_{e}}{N_{e}}
$$

Paso 13. Área proyectada de la placa $\left(\mathrm{A}_{1 \mathrm{p}}\right)$ :

$$
A_{1 p}=\left(L_{v}-D_{p}\right) \cdot L_{a}
$$

Paso 14. Factor de expansión calculado $(\phi)$ :

$$
\phi=\frac{A_{1}}{A_{1 p}}
$$

Paso 15. Diámetro equivalente hidráulico del canal $\left(D_{h}\right)$ :

$$
D_{h}=\frac{2 \cdot b}{\phi}
$$

Paso 16. Número de canales por pase $\left(\mathrm{N}_{\mathrm{cp}}\right)$ :

$$
N_{c p}=\frac{N_{t}-1}{2 \cdot N_{p}}
$$

Paso 17. Caudal másico por canal:

Fluido caliente $\left(\mathrm{m}_{\mathrm{cc}}\right)$ :

$$
m_{c c}=\frac{m_{c}}{N_{c p}}
$$

Fluido frío $\left(\mathrm{m}_{\mathrm{cf}}\right)$ :

$$
m_{c f}=\frac{m_{f}}{N_{c p}}
$$

Paso 18. Velocidad másica: 


\section{A. Pérez Sánchez et al.}

Fluido caliente $\left(\mathrm{G}_{\mathrm{c}}\right)$ :

$$
G_{c}=\frac{m_{c c}}{A_{f}}
$$

Fluido frío $\left(\mathrm{G}_{f}\right)$ :

$$
G_{f}=\frac{m_{c f}}{A_{f}}
$$

Paso 19. Número de Reynolds:

Fluido caliente $\left(\operatorname{Re}_{\mathrm{c}}\right)$ :

$$
\operatorname{Re}_{c}=\frac{G_{c} \cdot D_{h}}{\mu_{c}}
$$

Fluido frío $\left(\mathrm{Re}_{\mathrm{f}}\right)$ :

$$
\operatorname{Re}_{f}=\frac{G_{f} \cdot D_{h}}{\mu_{f}}
$$

Paso 20. Número de Prandtl:

Fluido caliente $\left(\operatorname{Pr}_{\mathrm{c}}\right)$ :

$$
\operatorname{Pr}_{c}=\frac{C p_{c} \cdot \mu_{c}}{k_{c}}
$$

Fluido frío $\left(\operatorname{Pr}_{\mathrm{f}}\right)$ :

$$
\operatorname{Pr}_{f}=\frac{C p_{f} \cdot \mu_{f}}{k_{f}}
$$

Paso 21. Coeficiente pelicular de transferencia de calor:

Fluido caliente $\left(\mathrm{h}_{\mathrm{c}}\right)$ :

$$
h_{c}=\frac{k_{c}}{D_{h}} \cdot C_{h} \cdot \operatorname{Re}_{c}^{n} \cdot \operatorname{Pr}_{c}^{1 / 3} \cdot\left(\frac{\mu_{c}}{\mu_{p}}\right)^{0,17}
$$

Fluido frío $\left(\mathrm{h}_{\mathrm{f}}\right)$ :

$$
h_{f}=\frac{k_{f}}{D_{h}} \cdot C_{h} \cdot \operatorname{Re}_{f}^{n} \cdot \operatorname{Pr}_{f}^{1 / 3} \cdot\left(\frac{\mu_{f}}{\mu_{p}}\right)^{0,17}
$$

Donde $\mathrm{C}_{\mathrm{h}} \mathrm{y} \mathrm{n}$ son parámetros que dependen del ángulo de la placa Chevron y del número de Reynolds, $\mathrm{y}$ se buscan en la Tabla 11.6, pág. 472, de (Kakaç et al., 2012). 
Paso 22. Coeficiente global de transferencia de calor limpio $\left(\mathrm{U}_{\mathrm{L}}\right)$ :

$$
U_{L}=\frac{1}{\frac{1}{h_{c}}+\frac{1}{h_{f}}+\frac{e_{p}}{k_{p}}}
$$

Paso 23. Coeficiente global de transferencia de calor sucio o de servicio $\left(\mathrm{U}_{\mathrm{S}}\right)$ :

$$
U_{s}=\frac{1}{\frac{1}{U_{L}}+R_{c}+R_{f}}
$$

Paso 24. Factor de limpieza (FL):

$$
F L=\frac{U_{s}}{U_{L}}
$$

Paso 25. Carga de calor para la superficie limpia (QL):

$$
Q_{L}=U_{L} \cdot A_{e} \cdot \Delta T_{m}
$$

Paso 26. Carga de calor para la superficie sucia $\left(\mathrm{Q}_{\mathrm{s}}\right)$ :

$$
Q_{s}=U_{s} \cdot A_{e} \cdot \Delta T_{m}
$$

Paso 27. Factor de seguridad (Fs):

$$
F s=\frac{Q_{s}}{Q}
$$

Paso 28. Porcentaje de sobrediseño de superficie (PSS):

$$
P S S=100 \cdot U_{L} \cdot\left(R_{c}+R_{f}\right)
$$

\subsubsection{Caída de presión}

La caída de presión total de fluidos que circulan por un ICP está compuesta por dos tipos de caídas de presión: 1) la caída de presión friccional en los canales y 2) la caída de presión en los puertos (Kakaç et al., 2012). A continuación, se muestra la metodología empleada para determinar este parámetro para cada fluido.

Paso 29. Coeficiente de fricción: 


\section{A. Pérez Sánchez et al.}

Fluido caliente $\left(f_{\mathrm{c}}\right)$ :

$$
f_{c}=\frac{K_{p}}{\operatorname{Re}_{c}^{m}}
$$

Fluido frío $\left(f_{f}\right)$ :

$$
f_{f}=\frac{K_{p}}{\operatorname{Re}_{f}^{m}}
$$

Donde $\mathrm{K}_{\mathrm{p}} \mathrm{y} \mathrm{m}$ son parámetros que dependen del ángulo de la placa Chevron y del número de Reynolds, y se buscan en la Tabla 11.6, pág. 472, de (Kakaç et al., 2012).

Paso 30. Caída de fricción friccional:

Fluido caliente $\left[\Delta \mathrm{P}_{\mathrm{f}(\mathrm{c})}\right]$ :

$$
\Delta P_{f(c)}=4 \cdot f_{c} \cdot \frac{L_{e f} \cdot N_{p}}{D_{h}} \cdot \frac{G_{c}^{2}}{2 \cdot \rho_{c}} \cdot\left(\frac{\mu_{c}}{\mu_{p}}\right)^{-0,17}
$$

Fluido frío $\left[\Delta \mathrm{P}_{\mathrm{f}(\mathrm{f})}\right]$ :

$$
\Delta P_{f(f)}=4 \cdot f_{f} \cdot \frac{L_{e f} \cdot N_{p}}{D_{h}} \cdot \frac{G_{f}^{2}}{2 \cdot \rho_{f}} \cdot\left(\frac{\mu_{f}}{\mu_{p}}\right)^{-0,17}
$$

Paso 31. Velocidad másica del puerto:

Fluido caliente $\left[\mathrm{G}_{\mathrm{p}(\mathrm{c})}\right]$ :

$$
G_{p(c)}=\frac{m_{c}}{\frac{\pi \cdot D_{p}^{2}}{4}}
$$

Fluido frío $\left[\mathrm{G}_{\mathrm{p}(\mathrm{f})}\right]$ :

$$
G_{p(f)}=\frac{m_{f}}{\frac{\pi \cdot D_{p}^{2}}{4}}
$$

Paso 32. Caída de presión en los conductos del puerto:

Fluido caliente $\left[\Delta \mathrm{P}_{\mathrm{p}(\mathrm{c})}\right]$ :

$$
\Delta P_{p(c)}=1,4 \cdot N_{p} \cdot \frac{G_{p(c)}^{2}}{2 \cdot \rho_{c}}
$$

Fluido frío $\left[\Delta \mathrm{P}_{\mathrm{p}(\mathrm{f})}\right]$ : 


$$
\Delta P_{p(f)}=1,4 \cdot N_{p} \cdot \frac{G_{p(f)}^{2}}{2 \cdot \rho_{f}}
$$

Paso 33. Caída de presión total:

Fluido caliente $\left[\Delta \mathrm{P}_{\mathrm{t}(\mathrm{c})}\right]$ :

$$
\Delta P_{t(c)}=\Delta P_{f(c)}+\Delta P_{p(c)}
$$

Fluido frío $\left[\Delta \mathrm{P}_{\mathrm{t}(\mathrm{f})}\right]$ :

$$
\Delta P_{t(f)}=\Delta P_{f(f)}+\Delta P_{p(f)}
$$

2.2.3. Efectividad de la transferencia de calor y número de unidades de transferencia

Se procedió a determinar los parámetros efectividad de la transferencia de calor $(\varepsilon)$ y el número de unidades de transferencia (NUT).

Paso 34. Tasa de capacidad calórica de cada corriente:

Fluido caliente $\left(\mathrm{C}_{\mathrm{c}}\right)$ :

$$
C_{c}=m_{c} \cdot C p_{c}
$$

Fluido frío $\left(\mathrm{C}_{\mathrm{f}}\right)$ :

$$
C_{f}=m_{f} \cdot C p_{f}
$$

Paso 35. Relación de la tasa de capacidad calórica $\left(\mathrm{C}^{*}\right)$ :

$$
C^{*}=\frac{C_{\min }}{C_{\max }}
$$

Paso 36. Efectividad de transferencia de calor del intercambiador $(\varepsilon)$ :

$$
\varepsilon=\frac{C_{c} \cdot\left(T_{1}-T_{2}\right)}{C_{\min } \cdot\left(T_{1}-t_{1}\right)}=\frac{C_{f} \cdot\left(t_{2}-t_{1}\right)}{C_{\min } \cdot\left(T_{1}-t_{1}\right)}
$$

Paso 37. Determinación del Número de Unidades de Transferencia (NUT):

$$
N U T=\frac{1}{1-C^{*}} \cdot \ln \left(\frac{1-\varepsilon \cdot C^{*}}{1-\varepsilon}\right)
$$

2.3. Evaluación de la influencia del incremento de dos variables sobre cuatro parámetros de evaluación del ICP

Nexo Revista Científica / Vol. 33, No. 01, pp. 01-21 / Junio 2020 


\section{A. Pérez Sánchez et al.}

Se llevaron a cabo dos estudios con el fin de evaluar la influencia del incremento de dos variables de entrada de la corriente de ácido acético, sobre determinados parámetros de operación y diseño del equipo. En el primer estudio se evaluará la influencia del aumento del caudal de alimentación del ácido acético (mc) sobre dos variables: 1) porcentaje sobrediseño de superficie (PSS), y 2) caída de presión del ácido acético $\left[\Delta \mathrm{P}_{\mathrm{t}(\mathrm{c})}\right]$, mientras que en el segundo estudio se determinará el efecto del incremento de la temperatura de entrada del ácido acético $\left(\mathrm{T}_{1}\right)$ sobre cuatro parámetros: 1) porcentaje sobrediseño de superficie (PSS), 2) caída de presión del ácido acético $\left.\left[\Delta \mathrm{P}_{\mathrm{t}(\mathrm{c})}\right], 3\right)$ efectividad de transferencia de calor del intercambiador ( $(\varepsilon)$ y 4) número de unidades de transferencia (NUT). En este caso, el caudal de alimentación del ácido acético se variará entre $10-80 \mathrm{~kg} / \mathrm{s}$, y la temperatura de entrada entre $60-130{ }^{\circ} \mathrm{C}$, manteniendo constantes los restantes parámetros de operación del proceso y del equipo. Se obtuvieron figuras que relacionan, desde el punto de vista gráfico, las variables dependientes o de salida con las independientes o de entrada.

\section{RESULTADOS Y DISCUSIÓN}

\subsection{Porcentaje de sobrediseño de superficie}

Seguidamente se muestran los resultados alcanzados de acuerdo con los pasos establecidos con anterioridad, hasta determinar el parámetro diseño porcentual superficial (DPS) del intercambiador de calor de placas.

Paso 1. Recopilación de los datos iniciales de las corrientes:

En la Tabla 1 se observan los valores de los parámetros iniciales de cada corriente.

Tabla 1. Valores de los parámetros iniciales de cada corriente establecidos en el Paso 1.

\begin{tabular}{lccc}
\hline Parámetro & Símbolo & Valor & Unidades \\
\hline Caudal másico ácido acético & $\mathrm{m}_{\mathrm{C}}$ & 24 & $\mathrm{~kg} / \mathrm{s}$ \\
Temperatura de entrada ácido acético & $\mathrm{T}_{1}$ & 80 & ${ }^{\circ} \mathrm{C}$ \\
Temperatura de salida ácido acético & $\mathrm{T}_{2}$ & 30 & ${ }^{\circ} \mathrm{C}$ \\
Caída de presión máxima permisible del ácido & $\Delta \mathrm{P}^{\prime}{ }_{(\mathrm{c})}$ & 100000 & $\mathrm{~Pa}$ \\
acético & & & \\
Factor de ensuciamiento ácido acético & $\mathrm{R}_{\mathrm{C}}$ & $0,0002^{\S}$ & $\mathrm{m}^{2} \cdot \mathrm{K} / \mathrm{W}$ \\
Temperatura de entrada etilenglicol & $\mathrm{T}_{1}$ & 5 & ${ }^{\circ} \mathrm{C}$ \\
Temperatura de salida etilenglicol & $\mathrm{t}_{2}$ & 25 & ${ }^{\circ} \mathrm{C}$ \\
Caída de presión máxima permisible del etilenglicol & $\Delta \mathrm{P}^{\prime}{ }^{\mathrm{c})}$ & 100000 & ${ }^{\mathrm{Pa}}$ \\
Factor de ensuciamiento etilenglicol & $\mathrm{R}_{\mathrm{f}}$ & $0,0002^{\S}$ & $\mathrm{m}^{2} \cdot \mathrm{K} / \mathrm{W}$ \\
\hline
\end{tabular}

§Tomado de (Sinnott, 2005)

Fuente: Elaboración propia

Paso 2. Recopilación de los datos iniciales del intercambiador de calor de placas

La siguiente tabla (Tabla 2) expone los datos iniciales del intercambiador de calor de placas, los cuales fueron definidos en el problema. 
Tabla 2. Datos iniciales del intercambiador de calor de placas

\begin{tabular}{lccc}
\hline Parámetro & Símbolo & Valor & Unidades \\
\hline Espesor de las placas & $\mathrm{e}_{\mathrm{p}}$ & 0,0007 & $\mathrm{~m}$ \\
Ángulo de la placa Chevron & $\beta$ & 50 & $\mathrm{o}$ \\
Número total de placas & $\mathrm{N}_{\mathrm{t}}$ & 107 & - \\
Factor de expansión & $\phi$ & 1,25 & - \\
Número de pases & $\mathrm{N}_{\mathrm{p}}$ & 1 & - \\
Área total efectiva & $\mathrm{A}_{\mathrm{e}}$ & 115 & $\mathrm{~m}^{2}$ \\
Diámetro de todos los puertos & $\mathrm{D}_{\mathrm{p}}$ & 0,2 & $\mathrm{~m}$ \\
Longitud del paquete de placas comprimido & $\mathrm{L}_{\mathrm{p}}$ & 0,44 & $\mathrm{~m}$ \\
Distancia vertical entre puertos & $\mathrm{L}_{\mathrm{v}}$ & 1,55 & $\mathrm{~m}$ \\
Distancia horizontal entre puertos & $\mathrm{L}_{\mathrm{h}}$ & 0,43 & $\mathrm{~m}$ \\
Amplitud efectiva del canal & $\mathrm{L}_{\mathrm{a}}$ & 0,63 & $\mathrm{~m}$ \\
Conductividad térmica del material de la placa & $\mathrm{k}_{\mathrm{p}}$ & 16,5 & $\mathrm{~W} / \mathrm{mK}$ \\
\hline Funte: Elaboráín propia & & &
\end{tabular}

Fuente: Elaboración propia

Paso 3. Temperatura media de cada corriente:

- Ácido acético:

$$
\bar{T}=\frac{T_{1}+T_{2}}{2}=\frac{80+30}{2}=55^{\circ} \mathrm{C}
$$

- Etilenglicol:

$$
\bar{t}=\frac{t_{1}+t_{2}}{2}=\frac{5+25}{2}=15^{\circ} \mathrm{C}
$$

Paso 4. Propiedades físicas de los fluidos a la temperatura media determinada en el paso anterior.

De acuerdo con (Perry, 2008), tanto el ácido acético como el etilenglicol presentan los siguientes valores de las propiedades físicas (Tabla 3):

Tabla 3. Propiedades físicas de ambos fluidos de trabajo determinadas en el Paso 4

\begin{tabular}{lccc}
\hline Propiedad & Ácido acético & Etilenglicol & Unidades \\
\hline Calor especifico & 2182,81 & 2352,25 & $\mathrm{~J} / \mathrm{kg} . \mathrm{K}$ \\
Viscosidad & $7,471 \cdot 10^{-4}$ & $2,625 \cdot 10^{-2}$ & $\mathrm{Pa.s}$ \\
Conductividad térmica & 0,1538 & 0,2521 & $\mathrm{~W} / \mathrm{m} \cdot \mathrm{K}$ \\
Densidad & 1010,95 & 1117,32 & $\mathrm{~kg} / \mathrm{m}^{3}$ \\
\hline Fuente: Elaboración propia & & &
\end{tabular}

Paso 5. Cálculo de la carga de calor (Q):

Se emplearan los datos de la corriente caliente (ácido acético) para determinar Q ya que son los que se encuentran completos. Así:

$$
\begin{aligned}
& Q=m_{C} \cdot C p_{C} \cdot\left(T_{1}-T_{2}\right)=24 \cdot 2182,81 \cdot(80-30) \\
& Q=2619372 W
\end{aligned}
$$




\section{A. Pérez Sánchez et al.}

Luego se procede a determinar el flujo másico de etilenglicol necesario para cumplir con la demanda térmica del proceso. De esta manera, se parte de:

$$
Q=m_{f} \cdot C p_{f} \cdot\left(t_{2}-t_{1}\right)
$$

Luego se despeja y determina $\mathrm{m}_{\mathrm{f}}$ :

$$
\begin{aligned}
& m_{f}=\frac{Q}{C p_{f} \cdot\left(t_{2}-t_{1}\right)}=\frac{2619372}{2352,25 \cdot(25-5)} \\
& m_{f}=55,68 \frac{\mathrm{kg}}{\mathrm{s}}
\end{aligned}
$$

La Tabla 4 muestra los valores de los parámetros determinados en los pasos 6-16.

Tabla 4. Resultados de los parámetros determinados en los pasos 6-16

\begin{tabular}{lccc}
\hline Parámetro & Símbolo & Valor & Unidades \\
\hline Diferencia media verdadera de temperatura & $\Delta \mathrm{T}_{\mathrm{m}}$ & 38,07 & ${ }^{\circ} \mathrm{C}$ \\
Número efectivo de placas & $\mathrm{N}_{\mathrm{e}}$ & 105 & \\
Longitud de flujo efectiva entre los puertos verticales & $\mathrm{L}_{\mathrm{ef}}$ & 1,55 & $\mathrm{~m}$ \\
Paso de la placa & $\mathrm{p}$ & 0,0041 & $\mathrm{~m}$ \\
Abertura promedio del canal de flujo & $\mathrm{b}$ & 0,0034 & $\mathrm{~m}$ \\
Área de flujo de un canal & $\mathrm{A}_{\mathrm{f}}$ & 0,0021 & $\mathrm{~m}^{2}$ \\
Área de transferencia de calor de una placa simple & $\mathrm{A}_{1}$ & 1,095 & $\mathrm{~m}^{2}$ \\
Área proyectada de la placa & $\mathrm{A}_{\mathrm{lp}}$ & 0,8505 & $\mathrm{~m}^{2}$ \\
Factor de expansión calculado & $\phi$ & 1,287 & \\
Diámetro equivalente hidráulico del canal & $\mathrm{D}_{\mathrm{h}}$ & 0,0053 & $\mathrm{~m}$ \\
Número de canales por pase & $\mathrm{N}_{\mathrm{cp}}$ & 53 & \\
\hline Funente: Elaborá́n propia & & &
\end{tabular}

La Tabla 5 exhibe los resultados obtenidos al determinar los parámetros de los pasos 17-20

Tabla 5. Resultados de los parámetros determinados en los pasos 17-20

\begin{tabular}{lccc}
\hline Parámetro & Ácido acético & Etilenglicol & Unidades \\
\hline Caudal másico por canal & 0,45 & 1,05 & $\mathrm{~kg} / \mathrm{s}$ \\
Velocidad másica & 214,29 & 500 & $\mathrm{~kg} / \mathrm{m}^{2} . \mathrm{s}$ \\
Número de Reynolds & 1520,19 & 100,95 & - \\
Número de Prandtl & 10,60 & 244,93 & - \\
\hline Fuente: Elaboración propia & &
\end{tabular}

Fuente: Elaboración propia

Paso 21. Coeficiente pelicular de transferencia de calor:

- Ácido acético $\left(\mathrm{h}_{\mathrm{c}}\right)$ :

$$
h_{c}=\frac{k_{c}}{D_{h}} \cdot C_{h} \cdot \operatorname{Re}_{c}^{n} \cdot \operatorname{Pr}_{c}^{1 / 3} \cdot\left(\frac{\mu_{c}}{\mu_{p}}\right)^{0,17}
$$


Dónde $\mathrm{Ch}=0,130$ y n $=0,732$ según Tabla 11.6, pág. 472, (Kakaç et al., 2012). También se asume que $\mu_{\mathrm{c}}$ $=\mu_{\mathrm{p}}=7,471 \cdot 10^{-4}$ (Kakaç et al., 2012). Entonces:

$$
\begin{aligned}
& h_{c}=\frac{0,1538}{0,0053} \cdot 0,130 \cdot(1520,19)^{0,732} \cdot(10,60)^{1 / 3} \cdot\left(\frac{7,471 \cdot 10^{-4}}{7,471 \cdot 10^{-4}}\right)^{0,17} \\
& h_{c}=1768,18 \frac{W}{m^{2} \cdot K}
\end{aligned}
$$

- Etilenglicol $\left(\mathrm{h}_{\mathrm{f}}\right)$ :

$$
h_{f}=\frac{k_{f}}{D_{h}} \cdot C_{h} \cdot \operatorname{Re}_{f}^{n} \cdot \operatorname{Pr}_{f}^{1 / 3} \cdot\left(\frac{\mu_{f}}{\mu_{p}}\right)^{0,17}
$$

Dónde $\mathrm{C}_{\mathrm{h}}=0,291$ y $\mathrm{n}=0,591$ según Tabla 11.6, pág. 472, (Kakaç et al., 2012). También se asume que $\mu_{\mathrm{f}}$ $=\mu_{\mathrm{p}}=2,625 \cdot 10^{-2}$ (Kakaç et al., 2012). Luego:

$$
\begin{aligned}
& h_{f}=\frac{0,2521}{0,0053} \cdot 0,291 \cdot(100,95)^{0,591} \cdot(244,93)^{1 / 3} \cdot\left(\frac{2,625 \cdot 10^{-2}}{2,625 \cdot 10^{-2}}\right)^{0,17} \\
& h_{f}=1323,93 \frac{W}{m^{2} \cdot K}
\end{aligned}
$$

La Tabla 6 expone los valores de los parámetros calculados en los pasos 22-28.

Tabla 6. Resultados de los parámetros determinados en los pasos 22-28

\begin{tabular}{|l|c|c|c|}
\hline Parámetro & Símbolo & Valor & Unidades \\
\hline Coeficiente global de transferencia de calor limpio & $\mathrm{U}_{\mathrm{L}}$ & 733,54 & $\mathrm{~W} / \mathrm{m}^{2} . \mathrm{K}$ \\
\hline $\begin{array}{l}\text { Coeficiente global de transferencia de calor sucio o de } \\
\text { servicio }\end{array}$ & $\mathrm{U}_{\mathrm{s}}$ & 567,13 & $\mathrm{~W} / \mathrm{m}^{2} . \mathrm{K}$ \\
\hline Factor de limpieza & FL & 0,77 & - \\
\hline Carga de calor para la superficie limpia & $\mathrm{Q}_{\mathrm{L}}$ & 3211474,79 & $\mathrm{~W}$ \\
\hline Carga de calor para la superficie sucia & $\mathrm{Q}_{\mathrm{s}}$ & 2482923,49 & $\mathrm{~W}$ \\
\hline Factor de seguridad & Fs & 0,947 & - \\
\hline Porcentaje de sobrediseño de superficie & PSS & 29,34 & $\%$ \\
\hline
\end{tabular}

Fuente: Elaboración propia

De acuerdo con los resultados mostrados en la Tabla 6, el ICP propuesto se encuentra sobrediseñado en aproximadamente un $29 \%$, mientras que el factor de seguridad calculado puede considerarse de adecuado (Kakaç et al., 2012).

\subsection{Caída de presión}

A continuación se procederá a determinar los coeficientes de fricción definidos por las ecuaciones (33) y (34), correspondientes al paso 29. En consecuencia:

Paso 29. Coeficiente de fricción:

Nexo Revista Científica / Vol. 33, No. 01, pp. 01-21 / Junio 2020 


\section{A. Pérez Sánchez et al.}

- Ácido acético $\left(\mathrm{f}_{\mathrm{c}}\right)$ :

$$
f_{c}=\frac{K_{p}}{\operatorname{Re}_{c}^{m}}
$$

Donde $\mathrm{K}_{\mathrm{p}}=0,772$ y $\mathrm{m}=0,161$ según Tabla 11.6, pág. 472, de (Kakaç et al., 2012). Luego:

$$
f_{c}=\frac{K_{p}}{\operatorname{Re}_{c}^{m}}=\frac{0,772}{(1520,19)^{0,161}}=0,237
$$

- Etilenglicol $\left(\mathrm{f}_{f}\right)$ :

$$
f_{f}=\frac{K_{p}}{\operatorname{Re}_{f}^{m}}
$$

Donde $\mathrm{K}_{\mathrm{p}}=11,251$ y $\mathrm{m}=$ 0,631 según Tabla 11.6, pág. 472, de (Kakaç et al., 2012). Entonces:

$$
f_{f}=\frac{K_{p}}{\operatorname{Re}_{f}^{m}}=\frac{11,251}{(100,95)^{0,631}}=0,612
$$

La Tabla 7 define los resultados obtenidos para los parámetros determinados en los pasos 30-33. Para el caso de las ecuaciones (35) y (36), los términos $\left(\mu_{\mathrm{c}} / \mu_{\mathrm{p}}\right)^{-0,17}$ y $\left(\mu_{\mathrm{f}} / \mu_{\mathrm{p}}\right)^{-0,17}$ presentes en las mismas se toman igual a uno (Kakaç et al., 2012).

Tabla 7. Resultados de los parámetros determinados en los pasos 30-33

\begin{tabular}{lccc}
\hline Parámetro & Ácido acético & Etilenglicol & Unidades \\
\hline Caída de fricción friccional & 6296,57 & 80089,70 & $\mathrm{~Pa}$ \\
Velocidad másica del puerto & 764,33 & 1773,25 & $\mathrm{~kg} / \mathrm{m}^{2} . \mathrm{s}$ \\
Caída de presión en los conductos del puerto & 404,52 & 1969,97 & $\mathrm{~Pa}$ \\
Caída de presión total & 6701,09 & 82059,67 & $\mathrm{~Pa}$ \\
\hline Fuente: Elaboración propia &
\end{tabular}

Según se puede observar en la Tabla 7, la caída de presión de tanto la corriente de ácido acético (6,70 kPa) como la de etilenglicol $(82,06 \mathrm{kPa})$ no sobrepasan la caída de presión máxima permisible establecida para el proceso para ambas corrientes $(100 \mathrm{kPa})$, lo cual quiere decir que el intercambiador de calor propuesto se comportará favorablemente con respecto a este parámetro para ambos fluidos.

3.3. Efectividad de la transferencia de calor y número de unidades de transferencia

Paso 34. Tasa de capacidad calórica de cada corriente:

- Ácido acético:

$$
C_{c}=m_{c} \cdot C p_{c}=24 \cdot 2182,81=52387,44 \frac{W}{K}
$$


- Etilenglicol:

$$
C_{f}=m_{f} \cdot C p_{f}=55,68 \cdot 2352,25=130973,28 \frac{W}{K}
$$

De esta manera, $\mathrm{C}_{\min }=\mathrm{C}_{\mathrm{c}} \mathrm{y} \mathrm{C}_{\max }=\mathrm{C}_{\mathrm{f}}$

Paso 35. Relación de la tasa de capacidad calórica:

$$
C^{*}=\frac{C_{\min }}{C_{\max }}=\frac{52387,44}{130973,28}=0,40
$$

Paso 36. Efectividad de transferencia de calor del intercambiador:

$$
\varepsilon=\frac{C_{c} \cdot\left(T_{1}-T_{2}\right)}{C_{\min } \cdot\left(T_{1}-t_{1}\right)}=\frac{\left(T_{1}-T_{2}\right)}{\left(T_{1}-t_{1}\right)}=\frac{80-30}{80-5}=0,667
$$

Paso 37. Determinación del Número de Unidades de Transferencia (NUT):

$$
\begin{aligned}
& N U T=\frac{1}{1-C^{*}} \cdot \ln \left(\frac{1-\varepsilon \cdot C^{*}}{1-\varepsilon}\right) \\
& N U T=\frac{1}{1-0,40} \cdot \ln \left(\frac{1-0,667 \cdot 0,40}{1-0,667}\right) \\
& N U T=1,316
\end{aligned}
$$

Considerando el valor de $\varepsilon$ determinado, se puede concluir que el ICP propuesto transferirá calor en un $66,7 \%$ con respecto a la máxima transferencia de calor posible que se puede alcanzar en el sistema, lo cual puede considerarse de aceptable (Pisinger, 2018). Por su parte el ICP tendrá un valor de NUT de 1,316, que puede calificarse de pequeña y adecuada para las condiciones del proceso (Pisinger, 2018).

Tomando en cuenta los resultados obtenidos, se concluye que el ICP propuesto puede emplearse satisfactoriamente para llevar a cabo el proceso de transferencia de calor considerado.

3.4. Resultados de la evaluación de la influencia del incremento del caudal másico del ácido acético sobre los dos parámetros seleccionados

La figura 2 muestra los resultados gráficos obtenidos al evaluar la influencia del caudal másico del ácido acético sobre los dos parámetros de operación considerados. 


\section{A. Pérez Sánchez et al.}

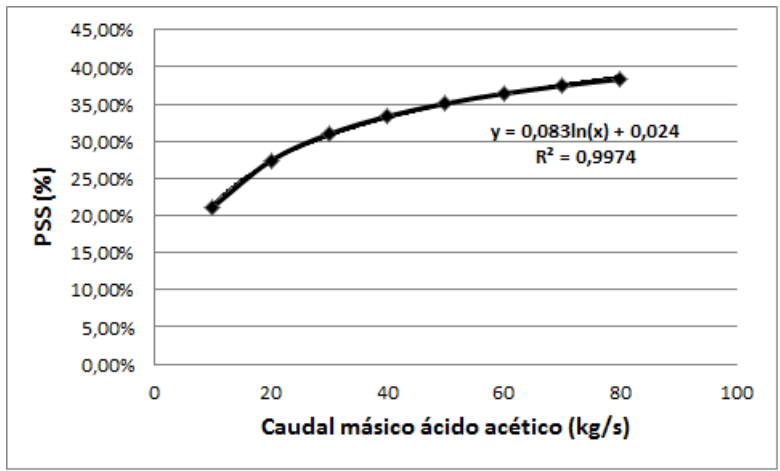

(a)

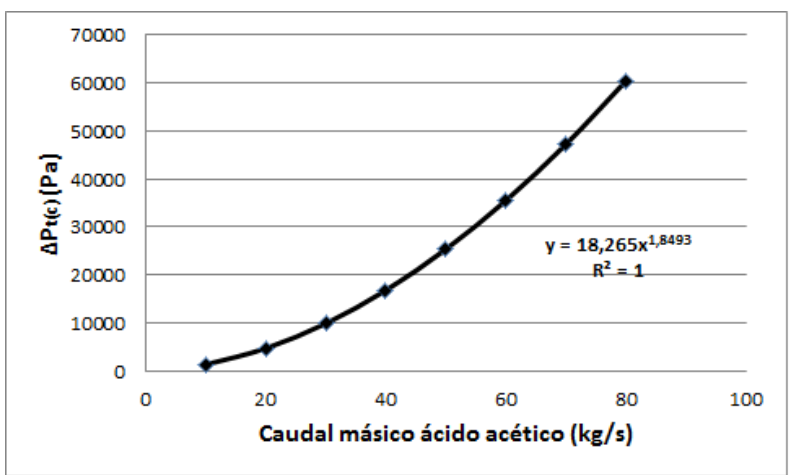

(b)

Figura 2. Influencia del incremento del caudal de alimentación sobre dos parámetros de operación del ICP. (a) Porcentaje de sobrediseño de superficie (PSS). (b) Caída de presión del ácido acético $\left[\Delta \mathrm{P}_{\mathrm{t}(\mathrm{c})}\right]$

Fuente: Elaboración propia

Según se puede observar en la Figura 2, los dos parámetros considerados se incrementan cuando aumenta el caudal másico de alimentación del ácido acético $\left(\mathrm{m}_{\mathrm{c}}\right)$.

En el caso del PSS, un incremento de mc aumenta también el caudal másico del etilenglicol $\left(\mathrm{m}_{\mathrm{f}}\right)$ necesario para cumplir con la demanda térmica del sistema, lo cual significa entonces un aumento del caudal másico por canal para cada fluido según las ecuaciones (16) y (17); un aumento de la velocidad másica de cada fluido según las ecuaciones (18) y (19); un incremento del número de Reynolds para ambos fluidos según las ecuaciones (20) y (21); un aumento de los coeficientes peliculares de transferencia de calor para cada fluido considerando las ecuaciones (24) y (25), y por último un incremento del coeficiente global de transferencia de calor limpio $\left(\mathrm{U}_{\mathrm{L}}\right)$ según la ecuación (26), el cual influye directamente en el incremento de la variable PSS según la ecuación (32).

Con respecto a la caída de presión del ácido acético, esta aumenta con un incremento de mc debido a que tanto la velocidad másica del ácido acético $(\mathrm{Gc})$ como la velocidad másica del puerto $\left[\mathrm{G}_{\mathrm{p}(\mathrm{c})}\right]$ se incrementan, las cuales son directamente proporcionales a la caída de presión friccional $\left[\Delta \mathrm{P}_{\mathrm{f}(\mathrm{c})}\right]$ y la caída de presión en los conductos del puerto $\left[\Delta \mathrm{P}_{\mathrm{p}(\mathrm{c})}\right]$, respectivamente. Esto es, si aumenta mc, aumenta tanto Gc como $\mathrm{G}_{\mathrm{p}(\mathrm{c})}$ según las ecuaciones (18) y (37), respectivamente, incrementando de esta manera tanto $\Delta \mathrm{P}_{\mathrm{f}(\mathrm{c})}$ como $\Delta \mathrm{P}_{\mathrm{p}(\mathrm{c})}$ de acuerdo con las ecuaciones (35) y (39).

3.5. Resultados de la evaluación de la influencia del incremento de la temperatura de entrada del ácido acético sobre los cuatro parámetros seleccionados

La figura 3 exhibe los gráficos alcanzados durante la evaluación del efecto del aumento de la temperatura de entrada del ácido acético, sobre los cuatro parámetros de operación tomados en cuenta.

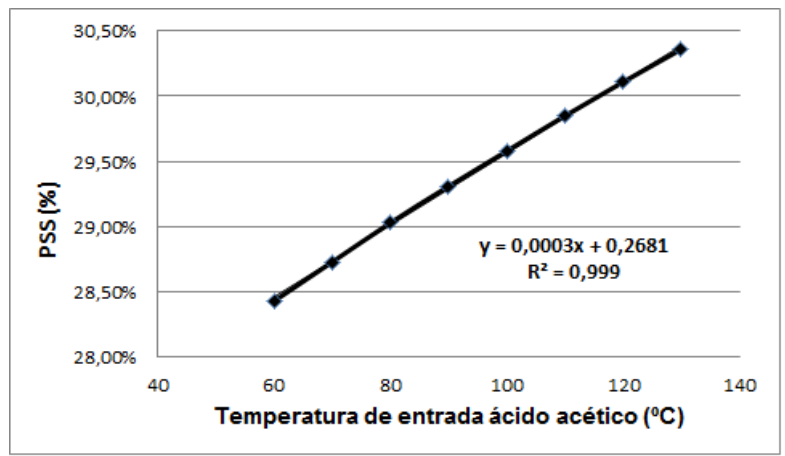

(a)

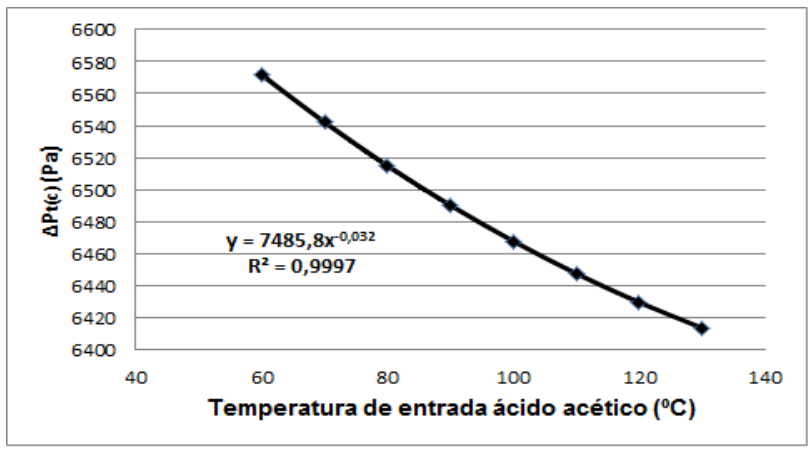

(b)

Nexo Revista Científica / Vol. 33, No. X1, pp. 01-21 / Junio 2020 


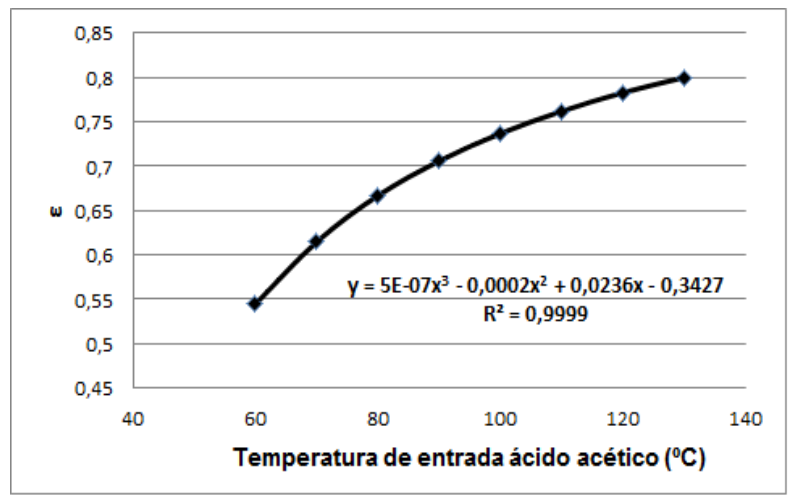

(c)

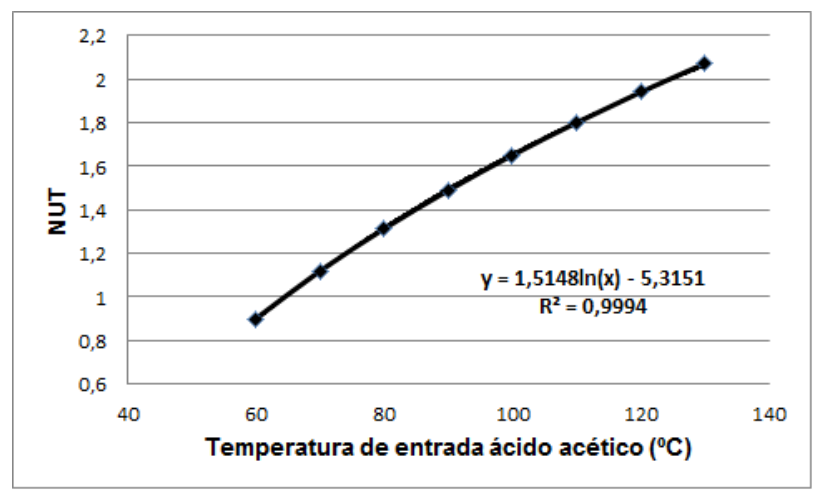

(d)

Figura 3. Influencia del aumento de la temperatura de entrada sobre cuatro parámetros de operación del ICP. (a) Porcentaje de sobrediseño de superficie (PSS). (b) Caída de presión del ácido acético $\left[\Delta \mathrm{P}_{\mathrm{t}(\mathrm{c})}\right]$. (c) Efectividad de transferencia de calor del intercambiador $(\varepsilon)$. (d) Número de unidades de transferencia (NUT).

Fuente: Elaboración propia.

De acuerdo con los resultados mostrados en la Figura 3, con excepción de la caída de presión las restantes variables aumentan cuando se incrementa la temperatura de entrada del ácido acético $\left(T_{1}\right)$.

En el caso del parámetro PSS (Figura 3a), un incremento de $\mathrm{T}_{1}$ provoca la reducción de la viscosidad del ácido acético $\left(\mu_{\mathrm{c}}\right)$, lo cual influye en el incremento del número de Reynolds según la ecuación (20). Esto provoca entonces un aumento del coeficiente pelicular de transferencia de calor del ácido acético $\left(\mathrm{h}_{\mathrm{c}}\right)$ según la ecuación (24), un incremento del coeficiente global de transferencia de calor limpio $\left(\mathrm{U}_{\mathrm{L}}\right)$ según la ecuación (26), y por último un aumento de PSS. Vale destacar que el incremento de $\mathrm{T}_{1}$ también incrementa el caudal másico necesario de etilenglicol $\left(\mathrm{m}_{\mathrm{f}}\right)$, por lo que se incrementa entonces el caudal másico por canal del etilenglicol $\left(\mathrm{m}_{\mathrm{cf}}\right)$ según la ecuación (17), la velocidad másica del etilenglicol $\left(\mathrm{G}_{\mathrm{f}}\right)$ de acuerdo con la ecuación (19), el número de Reynolds del etilenglicol $\left(\operatorname{Re}_{\mathrm{f}}\right)$ según la ecuación (21), el coeficiente pelicular de transferencia de calor del etilenglicol $\left(\mathrm{h}_{\mathrm{f}}\right)$ según la ecuación (25), el coeficiente global de transferencia de calor limpio $\left(\mathrm{U}_{\mathrm{L}}\right)$ de acuerdo con la ecuación (26) y por último la variable PSS según la ecuación (32).

Considerando la caída de presión del ácido acético $\left[\Delta \mathrm{P}_{\mathrm{t}(\mathrm{c})}\right]$ (Figura $3 \mathrm{~b}$ ), su reducción se debe a que a medida que aumenta $T_{1}$ disminuye el valor del factor de fricción según la ecuación (33), lo cual se considera suficiente para reducir $\Delta \mathrm{P}_{\mathrm{t}(\mathrm{c})}$ de forma proporcional de acuerdo con la ecuación (35).

La efectividad de transferencia de calor del intercambiador $(\varepsilon)$ aumenta con un incremento de $\mathrm{T}_{1}$ (Figura $3 c$ ) debido a que $T_{1}$ es directamente proporcional a $\varepsilon$ según la ecuación (46), es decir, si $T_{1}$ aumenta así lo hará de forma proporcional la variable $\varepsilon$.

Por último, el Número de Unidades de Transferencia (NUT) se incrementa con un aumento de $\mathrm{T}_{1}$ (Figura 3d), ya que el parámetro $C^{*}$ disminuye y $\varepsilon$ aumenta, lo cual influye de forma directa en el valor a obtener de NUT según la ecuación (47).

\section{CONCLUSIONES}

El ICP está un 29,34 \% sobrediseñado para las condiciones operacionales establecidas por el proceso. Tanto la caída de presión del ácido acético $(6,70 \mathrm{kPa})$ como la del etilenglicol $(82,06 \mathrm{kPa})$ se encuentran por debajo del valor máximo establecido por el proceso $(100 \mathrm{kPa})$.

El ICP tendrá una efectividad de transferencia de calor $(\varepsilon)$ de 0,667 y un Número de Unidades de Transferencia (NUT) de 1,316, los cuales se consideran adecuados. 


\section{A. Pérez Sánchez et al.}

El incremento del caudal de alimentación del ácido acético aumenta tanto el porcentaje de sobrediseño de superficie del ICP como la caída de presión del ácido acético.

El aumento de la temperatura de entrada del ácido acético incrementa tanto el porcentaje de sobrediseño de superficie, la efectividad de transferencia de calor del intercambiador como el número de unidades de transferencia, mientras que la caída de presión del ácido acético decrece.

El ICP propuesto puede ser empleado favorablemente para llevar a cabo el proceso de transferencia de calor considerado.

\section{REFERENCIAS}

Arsenyeva, O., Tovazhnyansky, L. L., Kapustenko, P., and Khavin, G. L (2009). Mathematical Modelling and Optimal Design of Plate-and-Frame Heat Exchangers. Chemical Engineering Transactions, 18, 791796. doi: 10.3303/CET0918129.

Arsenyeva, O. P., Tovazhnyansky, L. L., Kapustenko, P. O., and Khavin, G. L. (2011). Optimal design of plate-and-frame heat exchangers for efficient heat recovery in process industries. Energy, 36, 4588-4598. doi: 10.1016/j.energy.2011.03.022.

Cao, E. (2010). Heat transfer in process engineering. New York: McGraw-Hill.

Kakaç, S., Liu, H., and Pramuanjaroenkij, A. (2012). Heat Exchangers. Selection, Rating, and Thermal Design. Boca Raton: CRC Press.

Kumbhare, M. B., and Dawande, S. D. (2013). Performance Evaluation of Plate Heat Exchanger in Laminar and Turbulent Flow Conditions. International Journal of Chemical Sciences and Applications, 4 (1), 77-83.

Opatril, J., Havlík, J., Bartoš, O., and Dlouhý, T. (2016). An Experimental Assessment of the Plate Heat Exchanger Characteristics by Wilson Plot Method. Acta Polytechnica, 56 (5), 367-372. doi: 10.14311/AP.2016.56.0367.

Perry, R. H. (2008). Chemical Engineers' Handbook (8th ed.). New York: McGraw-Hill.

Pinto, J. M., and Gut, J. A. W. (2002). A Screening Method for the Optimal Selection of Plate Heat Exchanger Configurations. Brazilian Journal of Chemical Engineering, 19 (4), 433 - 439.

Pisinger, R. (2018). Optimization of an industrial heat exchanger by the minimalization of entropy. (Master's Thesis), Faculty of Mechanical Engineering, University of West Bohemia in Pilsen, Plzeň, Czech Republic.

Singh, S., and Jakhar, S. (2014). Performance Investigation of Plate Type Heat Exchanger (A Case Study). International Journal of Engineering Research and Applications, 4 (4), 127-131.

Sinnott, R. K. (2005). Chemical Engineering Design (4th ed., Vol. 6). Oxford: Elsevier ButterworthHeinemann.

Sreejith, K., Varghese, B., Das, D., Devassy, D., Harikrishnan, K., and Sharath, G. K. (2014). Design and Cost Optimization of Plate Heat Exchanger. Research Inventy: International Journal of Engineering and Science, 4 (10), 43-48. 
Torres, E., Díaz, E. J., Cedeño, M. P., Vargas, C. L., and Peralta, S. G. (2016). Heat transfer coefficients and efficiency loss in plate heat exchangers during the ammonia liquor cooling process. International Journal of Theoretical and Applied Mechanics, 1, 55-60.

Varona, B., González, R., Pérez, R. W., y Pérez, M. (2007). Análisis del intercambiador de calor de placas enfriador de mosto en la cervecería "Tínima”. Tecnología Química, XXVII (3), 61-67.

\section{SEMBLANZA DE LOS AUTORES}

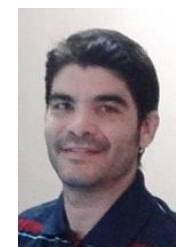

Amaury Pérez Sánchez: Obtuvo el grado de Ingeniero Químico en la Universidad de Camagüey, Cuba, donde actualmente es profesor instructor e investigador auxiliar. En estos momentos se encuentra estudiando un Máster en Biotecnología en el centro de Ingeniería Genética y Biotecnología de Camagüey. Trabaja en líneas de investigación vinculadas fundamentalmente con el diseño de equipos de transferencia de calor y masa, simulación y optimización de procesos y operaciones de la industria química mediante el empleo de simuladores, y la evaluación técnico-económica de procesos y plantas biotecnológicas.

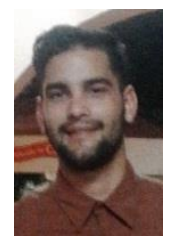

Eddy Javier Pérez Sánchez: Obtuvo el grado de Ingeniero Químico en la Universidad de Camagüey, Cuba. Se desempeña profesionalmente en la Empresa de Servicios Automotores S.A., en el Departamento de Dirección Comercial. Trabaja en líneas de investigación relacionadas con el diseño, evaluación y/o operación de equipos de transferencia de calor y masa, asi como también la simulación de procesos petroquímicos y biotecnológicos.

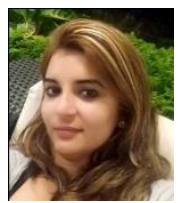

Elizabeth Ranero González. Obtuvo el grado de Ingeniero Químico en la Universidad de Camagüey, Cuba. Se desempeña profesionalmente como Profesor Instructor en la Universidad de Camagüey, Cuba. Su área de investigación abarca el diseño y evaluación de equipos y procesos de transferencia de calor y masa, evaluación termodinámica de procesos químicos, y la simulación de procesos y sistemas biotecnológicos. 